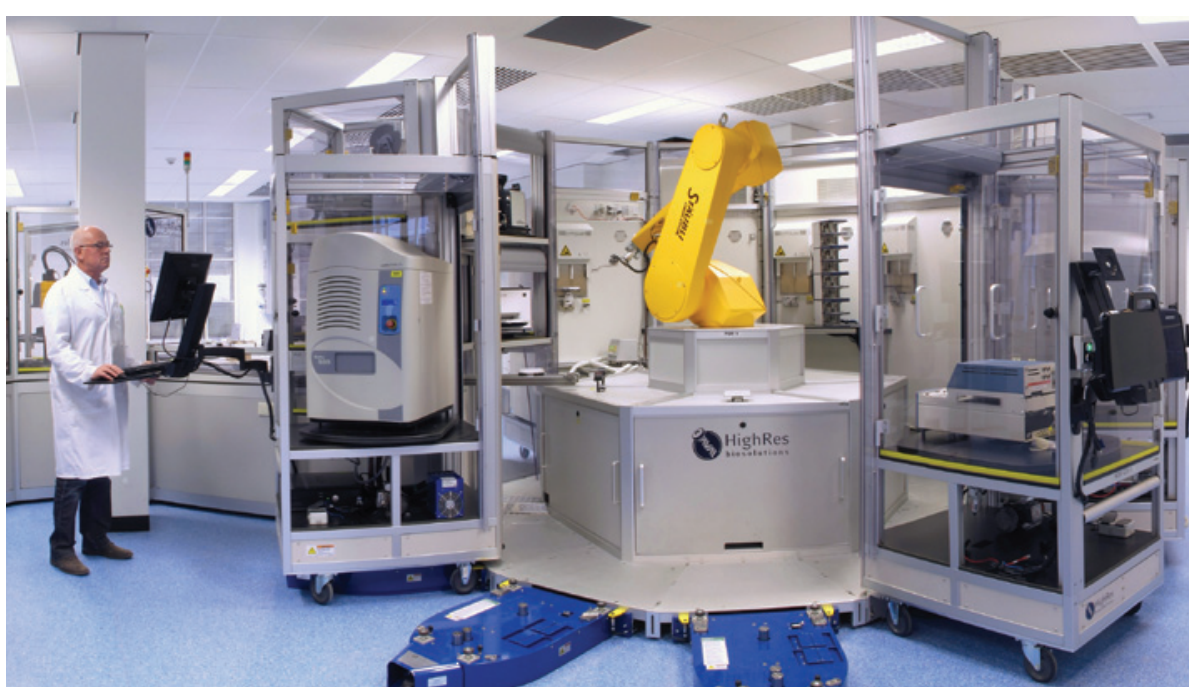

Robots in a facility in the Netherlands will screen molecules for biological activity.

\title{
DRUG DEVELOPMENT
}

\section{Europe bets on drug discovery}

\section{Proponents hope consortium will revive flagging industry.}

\section{BY MONYA BAKER}

$\mathrm{T}$ wo sites shuttered by the pharmaceutical giant Merck, one in Scotland and one in the Netherlands, will soon be humming again with the work of drug discovery. But the hum will not be business as usual. It will be the sound of a public-private consortium placing a high-stakes wager: a nearly $€ 200$-million (US\$271-million) bet that it can boost a languishing pharmaceutical sector by fusing academic innovation with industrial-scale screening, using robots to test chemicals for biological activity.

"If it really works, it might provide a future model to operate early drug discovery," says Jörg Hüser, a champion of the idea who works at Bayer Healthcare in Wuppertal, Germany. The scheme, announced on 7 February, is sponsored by the Europe's Innovative Medicine Initiative. The European Commission's Seventh Framework Programme is contributing $€ 80$ million to the venture, with the remaining $€ 116$ million coming from in-kind contributions from industry partners and regional governments.

Called the European Lead Factory, the consortium consists of 30 academic and corporate partners, and aims to fill company pipelines with promising drug candidates. The current dearth of candidates, Hüser believes, is due to gaps in the range of biological targets that industry is pursuing and in the libraries of compounds screened for activity against those targets.

To fill those gaps, the initiative will build and curate a collection of 500,000 molecules for screening, 300,000 of which will come from the seven large pharmaceutical partners. The rest - intended to cover classes of biologically active molecule that are poorly represented in current libraries - will be formulated and distributed in laboratory space in Newhouse, Scotland, that was closed by Merck in 2010.

\section{CORPORATE FEEDBACK}

Starting this July or August, the pharmaceutical partners will be able to use the library - including molecules from their competitors - in their own drug screens. Any academic group or company can also propose assays to test molecules in the library for biological activity. Lead-factory scientists will run these assays free of charge and confirm any promising results, working mainly in laboratory space closed by Merck in 2011 at Oss in the Netherlands. Follow-up work will be done at the University of Dundee in Scotland. Results will be provided confidentially to the groups that proposed the assays so that they can pursue further work and publications.

The hope is that members will build on the results to improve the molecules' biological properties and to gather evidence, such as tumour shrinkage, that the compounds may work as drugs. These molecules can then be licensed back to companies for further development. The scheme hopes to become selfsustaining by requiring milestone payments as drugs move from laboratory to clinic and from additional partnerships and screening services.

"I think this is completely new," says Ton Rijnders, co-director of the initiative and scientific director of the non-profit research enabler Top Institute Pharma in Leiden, the Netherlands. An effort launched by the US National Institutes of Health in 2004 called the Molecular Libraries Program (MLP) built a 400,000-compound library of commercially available molecules, but its goal was not to find potential drugs but to identify biological pathways that might make good drug targets.

The European initiative, by contrast, aims to propel drug development. Both the chemicals in the screening library and results from the assays will be proprietary. Factory partners will get first right of refusal in licensing deals.

Such restrictions are essential if a compound is ever going to make the long journey from a screening hit to a viable drug candidate, say experts. "To justify the subsequent investments you have to make in hit-to-drug lead programmes, it is crucial that you can patent the results and protect them," says Hüser.

But others say that early hits are just entry points; the real value is created in subsequent work. "I've never worried about the notion that the MLP was a public collection," says Hugh Rosen, whose work at the Scripps Research Institute Molecular Screening Center in La Jolla, California, led to a compound now in clinical trials for multiple sclerosis.

The complexity of the European scheme makes some outsiders sceptical of whether it will succeed. Aled Edwards leads the Structural Genomics Consortium at the University of Toronto, Canada, in which some drug companies contribute both chemical analysis and screening support, but all data are publicly available. Keeping data open and focusing on specific drug mechanisms makes his consortium's approach much simpler. "Intellectual-property deals, assays coming from everywhere, multi-institutional agreements. Wow, that's hard," he says. "But they are very smart people who have done this before. So if anyone can do it, they can." -

\section{CORRECTIONS}

The World View 'Scottish science is ready to go it alone' (Nature 493, 579; 2013) attributed a recent debate on Scottish independence to the Royal Society of Edinburgh when it should have been the Royal Society of Chemistry.

The News story 'Coffee rust regains foothold' (Nature 493, 587; 2013) gave the wrong year for the first major outbreak of the fungus in Costa Rica - it was 1989 not 1980. 